\title{
Resuspension processes in a wide range of particle sizes
}

\author{
Ana María Vidales ${ }^{1 *}$, Jesica Benito ${ }^{1}$, Rodolfo Uñac ${ }^{1}$, Luc Oger $^{2}$, and Irene Ippolito ${ }^{3}$ \\ ${ }^{1}$ INFAP, CONICET, Departamento de Física, Facultad de Ciencias Físico Matemáticas y Naturales, Universidad Nacional de San \\ Luis, Ejército de los Andes 950, D5700HHW, San Luis, Argentina \\ ${ }^{2}$ CNRS, IPR (Institut de Physique de Rennes), UMR 6251, Univ. Rennes, 35000 Rennes, France \\ ${ }^{3}$ Universidad de Buenos Aires, Facultad de Ingeniería, Grupo de Medios Porosos, Paseo Colón 850, 1063 Buenos Aires, Argentina
}

\begin{abstract}
Particulate matter offers a broad spectrum of phenomena, well known in the granular matter community. This fact is not only related to the individual or collective behaviour that grains can present depending on the external boundary conditions and/or internal interactions but, to the wide range of particle sizes. This feature confers the possibility of studying a particular phenomenon in different size scales. The main goal of this paper is to describe the detachment of particles from surfaces, where particles go from tens of microns to several millimetres, in a variety of scenarios. All the studied systems presented here have in common the need to determine the critical conditions to lose the equilibrium balance, initiating incipient motion. Solving the right time moment for particle resuspension, consideration of the local interactions between particle, surface and external applied forces can be achieved from different modelling approaches, from deterministic dynamics to statistical mechanics. This paper focuses on some of these approaches performed recently on grains through different size scales.
\end{abstract}

\section{Introduction}

The way in which micrometric particles to millimetre grains initiate their movement in a general dynamic process could be relevant to describe the setting up of many phenomena. For instance, the cleaning up of surfaces, the potential segregation of different natural particles, the entrainment of hazardous agents polluting the atmosphere, are examples where the determination of the movement onset is important to control the phenomenon.

Resuspension is a physical process that remobilizes particles already deposited on a surface under the action of an external agent, such as air currents, vibrations, rotations and impacts. In this framework, the basic system consisting in a spherical particle deposited on a flat surface and subjected to forces (probably one of the most familiars in physics) has helped to schematize and simplify the picturing of many complex phenomena as those cited above.

The forces intervening in each problem are set on from the assumptions made on the main microscopic interactions between particle/surface and external actions. This gives rise to different possible mechanisms for movement initiation, such as rolling, sliding and lift off. The prevalence of one mechanism over the other is not often easy to capture from experiments and both numerical and theoretical modelling are valuable approaches for strengthening the description of the problem.

The study of the movement initiation of micrometric particles deposited on a surface has captured the attention of researches in the fields of environmental sciences and nuclear safety since several decades. To provide some examples, mining operations generate an important amount of dust, which is directly related to the contamination of different geographic regions and emergence of toxicity problems [1,2]. Typically, particles of mineral dust are widespread over the sites where mining occurs because they are transported by agents like wind and/or basic operations as blasting, loading, transporting or crushing, which all have mechanical vibration in common [1-3].

As remarked earlier, another source of huge interest is the improvement of the security of nuclear facilities to control the eventual resuspension of radioactive particles after a decompression event (loss of vacuum accident), which inevitably produces a dangerous reentrainment of micrometric particles to the atmosphere [4-13].

Similarly, vibration as a perturbation of particle equilibrium has been analysed in many theoretical studies and engineering applications where the removal of small particles from surfaces in pursued [14]. Basic studies concerning the individual excitation of small spheres $(<1 \mathrm{~mm})$ deposited on a horizontal platform has been conducted in the past, where the role and measurement of adhesion forces were under focus [1517].

Along with vibration and aerodynamic methods, centrifugation is one of the most efficient techniques for micrometer particle removal and for measuring adhesion forces [10-21]. In the problem of particle centrifugation, the knowledge of the rotational dynamics of particles is fundamental for understanding

* corresponding author avidales@unsl.edu.ar

A video is available at https://doi.org/10.48448/rq1y-d273 
processes such those commonly used in industrial mixers and/or separators, fertilizer spreaders or flows under low gravity [22,23]. Indeed, although size separators are commonly vertical, some of the results reviewed in this work could be applied to the eventual adaption of those mechanical devices to horizontal size segregators $[22,24]$.

For larger grains $(\geq 200 \mu \mathrm{m})$, conditions for the initial destabilization of sand grains by the wind action are important to predict the transport of sandy matter in deserts [25]. Likewise, in granular matter manipulation stages, the mechanical collective vibration of grains, either horizontal or vertical, is also an important external perturbation giving rise to phenomena as segregation, convection cells, fluidization and many other interesting problems that have been extensively studied in the granular matter community [26-35].

For the case of coarse particles, it is still an open issue to determine the influence of the adhesion forces when particles interact with a surface made of a bed of other particles (rough surface) just before leaving the surface [37].

In conclusion, the dynamics involved in the movement initiation of a particle on a surface subjected to external perturbations is not simple, and the inclusion of all the parameters playing a role is not straightforward [38,39], even when only a threshold velocity for movement initiation is sought [40]. Thus, the subject is still open, even for particles of the millimeter size.

The aim of the present paper is to review some of the latest results of our work group related to the study of the critical conditions for movement initiation of a particle resting on a surface and subjected to an external action. In what follows, a series of experiments and simulations will be presented to illustrate the main features of the detachment process found for micrometric to millimeter particles on different type of surfaces.

\section{Destabilization of particles by aero- dynamic forces}

The most studied destabilization process of micrometric particles is the one performed by the action of aerodynamic forces [41]. In this particle size range, the complexity of the problem resides in the difficulties for measuring the microscopic adhesion forces that result from the particle/surface interaction through a blend of mechanical stress, chemical bonds and physical attractions. For example, knowledge of the particle size and the surface roughness is crucial for the calculation of the reduction and spread in the adhesion force [ 42 , 43]. Besides, other difficulties arise when the different flow conditions and aerodynamic forces have to be determined [41]. Consequently, theoretical approaches are required to model particle/surface interactions and, likewise, numerical models to predict the resuspension rates are key tools to validate the relevance of those theoretical approaches.

\subsection{Kinetic Monte Carlo method applied to resuspension}

The present section will briefly describe a numerical model that was recently developed to predict particle resuspension rates $[44,45]$. It is based on a Kinetic Monte Carlo method (KMC) and takes advantage of the analogy between the re-entrainment of aerosol particles from surfaces (activated by airflow) and the desorption process of molecules on a surface (activated by temperature) [46,47]. In other words, the resuspension of a particle is assumed to be caused by a stochastic process resulting from the balance between adhesion forces and the forces exerted by air flow close to the wall due to irregular bursts or turbulent eddies. We assume that the detachment rate equation follows the Arrhenius law [48]. As well known, MC methods have been used for decades as versatile tools to describe molecular processes on surfaces [49], and different extensions of this method have been proposed to take into account the kinetic mechanisms governing the resuspension of particles from a surface [9,47].

Assuming a N-particle system and considering only one possible type of transition, i.e., a deposited particle could resuspend incorporating to the gas stream, the probability $P(t) \mathrm{d} t$ of the system to perform a transition during the interval $(t ; t+\mathrm{d} t)$ is given by a Poisson process [50,51]:

$$
P(t) d t=R e^{-R d t}
$$

where $R=\sum_{i=0}^{N} r_{i}$ is the total transition rate for the whole system and $r_{i}$ is the resuspension transition rate for a single particle. The variable $t$, which is distributed following $P(t) \mathrm{d} t$, is replaced by a random number, $\xi$, uniformly distributed in the interval $(0,1)[50,51]$ :

$$
\Delta t=-\frac{\ln (\xi)}{R}
$$

In $\mathrm{KMC}$ simulation, Equation 2 gives the actual time in which the system performs a transition, setting the relationship between the "virtual" $\mathrm{MC}$ time and the "actual" time of the process.

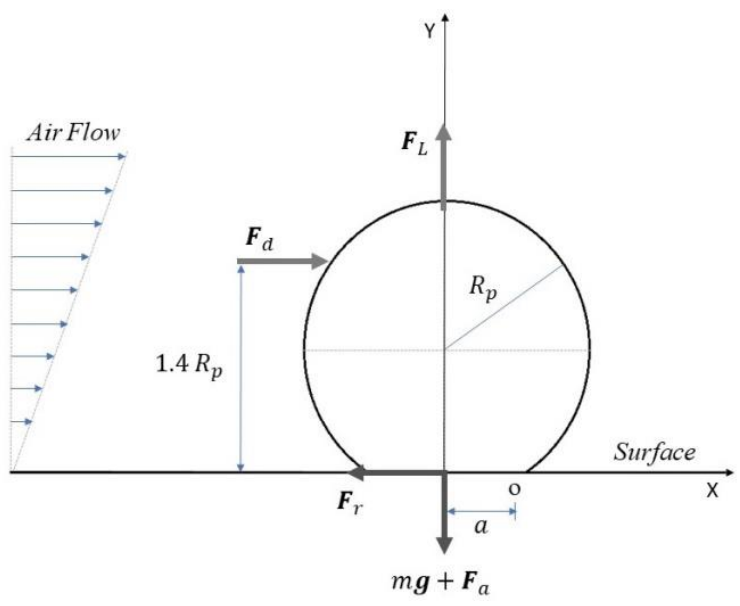

Fig. 1. Particle force diagram. The contact radius, $a_{0}$, comes from JKR theory [52]. 
In a typical simulation, an idealized monolayer deposit with $\mathrm{N}=10,000$ mono-sized particles with radius $R_{p}$ is considered, where the distance between particles is not relevant since they do not interact with each other, neither collide. Resuspension is considered as a process activated by turbulent air flow and particles are not allowed to be re-deposited or relocated in this model.

When a micro-particle is destabilized by airflow, the forces expected to be involved are schematized in Figure 1. These external actions are the aerodynamic forces of drag $\left(F_{d}\right)$ and lift $\left(F_{L}\right)$, the weight $(\mathrm{mg})$, the adhesion $\left(F_{a}\right)$ and the friction $\left(F_{r}\right)$ forces [53]. The three main possible mechanisms for particle take-off from a rough surface are: rolling, sliding, and pull-off. The corresponding critical equilibrium conditions are the following:

$$
\begin{array}{cc}
\left(1.4 R_{p}\right) F_{d}+a F_{L}>a\left(F_{a}+m g\right) & \text { (rolling) } \\
F_{d}>F_{r}=\mu\left[\left(F_{a}+m g\right)-F_{L}\right] & \text { (slide) } \\
F_{L}>\left(F_{a}+m g\right) & \text { (pull-off) }
\end{array}
$$

where the first equation is a moment balance and the second two are force balances.

Data coming from different sets of experiments in the last decades (mainly with spherical particles) and the contrast with different models, have proven with emphasis that the dominant initiation mechanism (for later resuspension) was rolling $[6,10,42,53]$. For that reason, most of the results presented here correspond to simulations where the rolling mechanism is considered as the only responsible for the movement initiation of particles before take-off. Thus, the transition rate, $r_{\text {roll }}$, for the only possible detachment process is defined from the moment balance condition as:

$$
r_{\text {roll }}=k \exp \left(-\frac{\left[a_{0}\left(F_{a}+F_{g}\right)-\left(1.4 R_{p} F_{d}+a_{0} F_{L}\right)\right]}{\left(1.4 R_{p} F_{d}+a_{0} F_{L}\right)}\right)
$$

where $k$ is a frequency factor interpreted as the maximum burst frequency in the removal process.

At the beginning of the simulation, the adhesion force is randomly assigned to each particle from a lognormal distribution, whose mean is in accordance with each of the respective interacting materials through JKR theory $[44,45,52]$. The reduction of adhesion due to the roughness of the substrate is taken into account through a dividing factor, $f_{r}$. The aerodynamic forces have been sampled from a Gaussian distribution, whose mean value (as most authors do) is assumed to correspond to the mean drag and lift on a sphere near a surface in simple shear flow in the predominantly viscous sub-layer, as most authors do $[42,10]$.

Typical experiments of resuspension are those where particles on a substrate are introduced in a wind tunnel and subjected to air flow with a linearly increased stream velocity, $U$. Thus, the recording of the resuspension rate is performed as a function of the friction velocity of the flow near the wall of the substrate (see Figure 2).

\subsection{Validation of the KMC mode}

Simulations with the transition rate given by Eq. 6 have been validated with experimental results from other authors $[10,42,45]$. Figure 2 shows the comparison of numerical and experimental results for $70 \mu \mathrm{m}$ stainlesssteel particles, 72 and $32 \mu \mathrm{m}$ glass particles on glass substrates [42]. Material properties are in Table 1.

Table 1. Simulation constants

\begin{tabular}{|c|c|c|c|}
\hline & SS 70 & Glass 72 & Glass 32 \\
\hline $\begin{array}{c}\text { Composite Young's } \\
\text { modulus }(\mathrm{Pa})\end{array}$ & $8.41 \mathrm{E} 10$ & $5.76 \mathrm{E} 10$ & $5.76 \mathrm{E} 10$ \\
\hline $\begin{array}{c}\text { Contact radius } a_{0} \\
(\mu \mathrm{m})\end{array}$ & 0.435 & 0.685 & 0.406 \\
\hline$F_{J K R}(N)$ & $2.47 \mathrm{E}-5$ & $6.59 \mathrm{E}-5$ & $3.02 \mathrm{E}-5$ \\
\hline$f_{r}$ & 100 & 200 & 43 \\
\hline
\end{tabular}

The description achieved by the KMC model is quite good in most of the inspected rage of friction velocities. The curves lie inside the range of experimental dispersion. In particular, the reduction factor, $f_{r}$, selected for each of the simulations (whose actual value is not determined) are close to the reported adhesion force reduction due to roughness $[10,42,54]$.

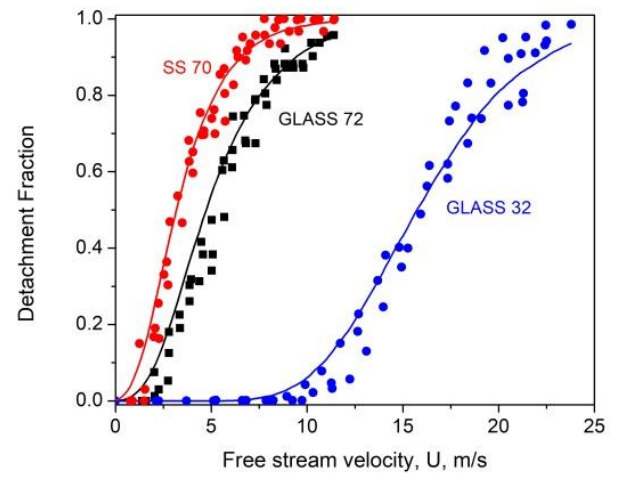

Fig. 2. Comparison between KMC results and experiments from [42]. Solid lines correspond to the simulation results. Symbols represent different repetitions of the experiments.

Comparing our model with the performance of other models $[6,10,41]$, the results here demonstrate that the present KMC methodology greatly improves the prediction capability of former numerical or theoretical descriptions. This goal is accomplished thanks to the stochastic nature introduced in the adhesion and aerodynamic forces entering the problem and in the statistical mechanics treatment applied, complementing former approaches $[10,43,53]$. This perspective is original since models up to now reduce the MC approaches to the resolution of integral equations for the resuspension rates, keeping always a deterministic spirit in the destabilization rules for movement initiation.

Finally, humidity effects are not introduced in the model because the experiments were conducted with a 
relative humidity around $25 \%$, indicating that the cohesive forces related to capillary effects were negligible [42].

\section{Destabilization of grains by vibration}

In this section, the main findings for the movement initiation of individual particles placed on a horizontal rough surface subject to a vertical sinusoidal vibration are presented as a result of a series of experiments, theoretical analysis, and numerical simulations performed in our work group in the last years $[55,56]$. The aim here is to determine the critical frequencyamplitude values of the vibration necessary to start the movement of at least half of the particles on the surface. These values are studied as a function of the properties of the mobile particles and the surface.

\subsection{Experiments}

To study the movement initiation of millimetre particles with different size and materials, the experimental set up schematically presented in Figure 3 was designed. The selected rough surface is mounted on the platform of the mechanical wave oscillator which can vibrate it vertically with a sinusoidal motion with controlled amplitude and frequency. Two methods are employed to record the movement, by a FULL HD camera recording and by direct observation with a loupe. The camera is connected to a PC for data recording and later processing.

The rough surfaces employed were fabricated by gluing glass beads of a given size on a flat circular plate using an EPOXI type adhesive. Two main different configurations were built. The multilayer configurations $(6 \mathrm{~cm}$ plate diameter) were made with $250 \mu \mathrm{m}$ and $500 \mu \mathrm{m}$ glass beads, respectively, with corresponding size dispersions of 44 and $85 \mu \mathrm{m}$. In the way the surfaces were manufactured, the resulting rough surface had between 2 and 3 layers of beads.

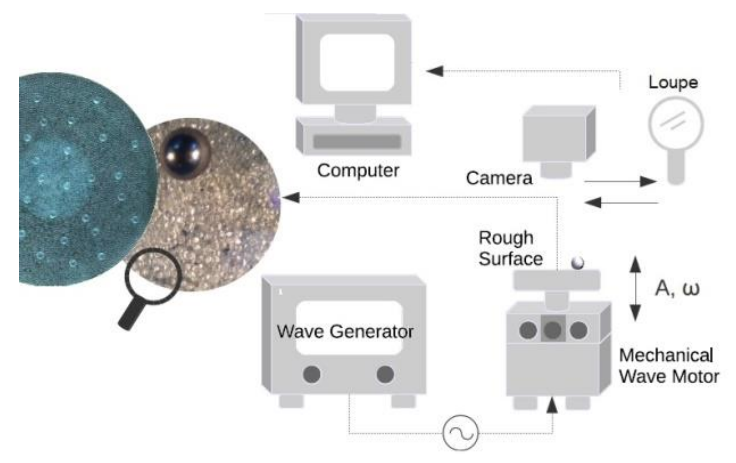

Fig. 3. Sketch of the experimental set up.

Similarly, the monolayer configurations were built with the same procedure of gluing but, controlling the coverage by randomly pouring a certain amount of glass grains of $1 \mathrm{~mm}$ (surface $\mathrm{S} 1$ ) and $2 \mathrm{~mm}$ (surface S2) on different plates with $18 \mathrm{~cm}$ in diameter, creating a quite homogeneous spatial distribution. Four different values for the coverage $\theta$ were produced: $50 \%, 66 \%, 70 \%$ and $82 \%$.

The particles deposited on the vibrating surfaces, correspond to glass beads with mean diameters of $(0.93$ $\pm 0.02),(1.98 \pm 0.04),(3.26 \pm 0.09)$, and $(3.98 \pm 0.03)$ $\mathrm{mm}$; and stainless-steel bearing balls of 2,3 , and 4 $\mathrm{mm}$. These grains were tried on the multilayer surfaces. On the other hand, the mobile spheres to be deposited on the monolayer surfaces for vibration were of two different materials, glass and Acrylonitrile Butadiene Styrene (ABS). They have different diameters equal to $3,6,7$ and $10 \mathrm{~mm}$ for glass particles and 3 and $5 \mathrm{~mm}$, for $\mathrm{ABS}$, all with relative size dispersion of the order of $1 \%$.

The procedure for a typical run is described as follows. Once the desired multilayer surface of $6 \mathrm{~cm}$ (or the monolayer one with $18 \mathrm{~cm}$ ) was mounted on the plate, 10 spheres (30 spheres for the monolayer case) of the selected sizes were carefully deposited on the rough substrate avoiding possible contact between them or with the lateral walls surrounding the plate. Then, the video recording is activated and the surface was subjected to a sinusoidal vibration at fixed amplitude, $A$. The frequency was increased continuously at a rate of $1 \mathrm{~Hz}$ every 6 seconds until all the grains initiated their movement. At that time, the vibration stops and the film was saved at the computer. Then, a new set of grains was distributed on the surface and a new run with a different amplitude value was started. The procedure was repeated five times for each surface and bead size.

For a given run, the critical frequency, $\omega_{c}$, was defined as the one at which half of the vertically vibrated grains had horizontally moved a displacement of the order of the grain radius. To determine $\omega_{c}$, each film is analysed with an open source image processing program ImageJ.

Although not critically important, humidity effects were present in most of the experiments. It was verified that inside a range of $30 \%$ to $50 \%$ of ambient humidity the reproducibility of the results was achieved.

Figure 4 shows typical results for the $500 \mu \mathrm{m}$ multi-layered substrate and mobile grains of glass, (a), and stainless steel, (b), with several size ratios, i.e., free sphere radius/surface sphere radius. The $\omega_{c}-A$ pairs separate the upper region, where at least $50 \%$ of the grains start their movement, from the lower part, where less than $50 \%$ of them are found in a condition of incipient movement.

The critical frequencies follow a clear exponential decay with the increment of the amplitude of the external sinusoidal excitation. All the completed experiments, i.e., both surfaces and both type of mobile grains, offered the same behaviour. This implies that the surface roughness created by the glued beads is such that its characteristic length is not governed by the size of the constituting beads, but rather by their geometrical arrangement created with the glue. In other words, the topography of the surface masks the influence of bead size. 

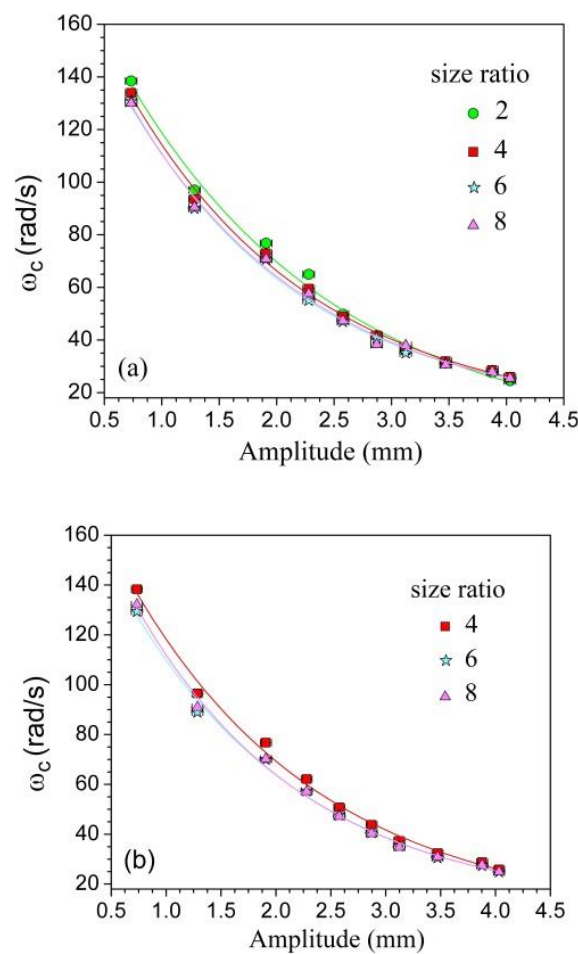

Fig. 4. Typical results for critical frequencies in the case of $500 \mu \mathrm{m}$ rough surface, (a) glass spheres, (b) stainless steel.

Concerning the influence of the mobile grain size, only a slight displacement of the data to higher frequencies for the smaller spheres is observed. Note that experiments with smaller $1 \mathrm{~mm}$ stainless-steel spheres were not performed due to static charge effects.

Finally, in agreement with other findings, the values for $\omega_{c}$ are much lower than those related to the elastic properties of particle material $\left(6 \times 10^{6} \mathrm{rad} / \mathrm{s}\right.$ or higher), thus, the simple regime of a forced oscillator has to be rethought to explain our present results [14], as seen in the next Section.

The exponential behaviour for $\omega_{c}$ vs. $A$ is again present for the case of monolayer substrates. Figure 5 shows two typical plots of the several cases studied, for the extreme coverage values $\theta=50 \%$ and $\theta=82 \%$ and mobile glass spheres. The sizes of the particles are indicated along with the corresponding surfaces used in each experiment. The values for the critical frequencies are again below those expected for the elastic properties of the particles.

When exponential fittings of the form $\omega_{c}(A)=$ $\omega_{c}(0) e^{-\gamma A}$ are performed on the respective curves, (those for the extreme cases are the curves drawn in Figure 5), the critical frequency for $A \rightarrow 0$ results to be lower as the size of the particle is greater, in agreement with the general behaviour of our results. On the other hand, the decaying constant $\gamma$ has the same value for all the cases studied, fluctuating around 0.19, independently of the surface or the mobile grain material.

\subsection{Theoretical approach}

In this Section the theoretical approach developed to explain the exponential-type behaviour found in the experiments will be briefly presented. The detailed procedure can be found in [55].
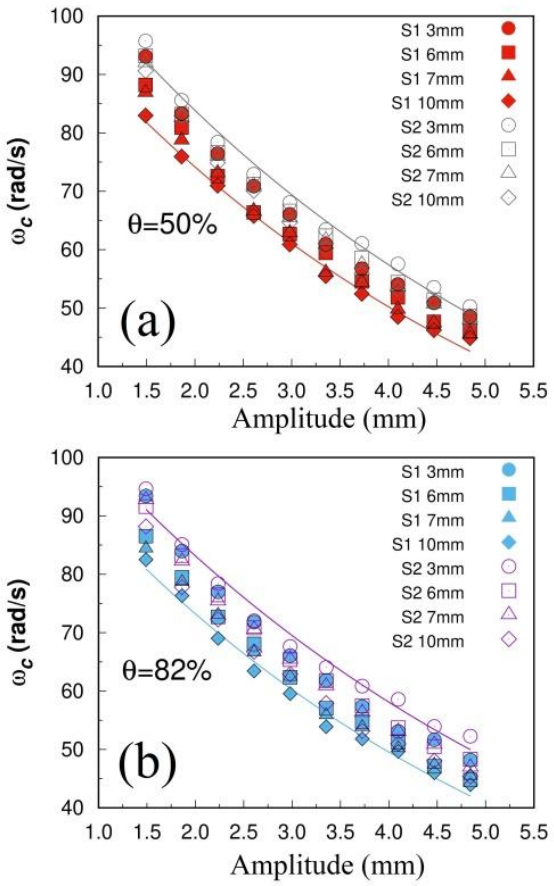

Fig. 5. Typical results for critical frequencies for glass spheres on both surfaces at extreme coverage values.

The model is based on the picture of a particle attached to a vibrating surface through a spring representing the effective interactions between particle and surface, arising from inter-molecular and capillary forces, which act (when combined) like an elastic bond or spring with stiffness $k$ [14]. Both forces increase with particle radius and are still important when compared with gravitational forces, even for particles with sizes of a few millimetres [57,58]. Although the relative humidity values at which experiments are performed are not very high, capillary bridges between particles and surface may be present, requiring capillary forces to be considered in the problem [59].

In the model, an external harmonic force is exerted by the vibrating bed on the particle via the spring. The vibration has amplitude $A$ and frequency $\omega / 2 \pi$ (inset in Figure 6). Using standard analysis for damped harmonic oscillations and considering that the applied experimental force is varied by the frequency change of the excitation, the amplitude seen by the sphere is:

$$
A^{*}(\omega)=\frac{A \omega^{2}}{\sqrt{\left(\omega_{0}^{2}-\omega^{2}\right)^{2}+(\gamma \omega)^{2}}}
$$

where $\omega_{0}$ and $\gamma$ are the natural frequency of the system and the damping constant for the elastic bond between particle and surface, respectively. A value of $200 \mathrm{~s}^{-1}$ was adopted for $\gamma$, which is (as seen below) of the order of the natural frequencies entering Eq. (7), thus modelling a lightly damped harmonic oscillator. 
Nonetheless, results demonstrated to be almost independent for $\gamma$ from $10^{2}$ to $10^{3} \mathrm{~s}^{-1}$.

The frequency $\omega_{0}$ was considered to depend on the excitation amplitude A. This is based on two reasons. On the one hand, quantifying the elastic properties of the bond between particle and surface is difficult and some assumptions for the estimation have to be done. On the other hand, authors found that the stiffness of a spherical particle is not constant but increases with particle deformation [14]. Here, the critical acceleration for detachment decreases as $A$ increases [55], so does the deformation of the particle. Thus, it is expected that $k$ (so $\omega_{0}$ ) decreases with $A$. Given the experimental behaviour found for $\omega_{c}$ vs. $A$, an exponential decay of the natural frequency $\omega_{0}$ with $A$ is proposed as a reasonable trend, consonant with [60].

From Equation (7) and assumptions above, the magnitude of the force needed for spring breaking can be calculated for different values of $A$, and, thus, the corresponding critical frequencies, $\omega_{c}$, can be obtained. Figure 6 compares experiments and theoretical approach for one of the tested cases, for $1 \mathrm{~mm}$ glass spheres over $250 \mu \mathrm{m}$ surface.

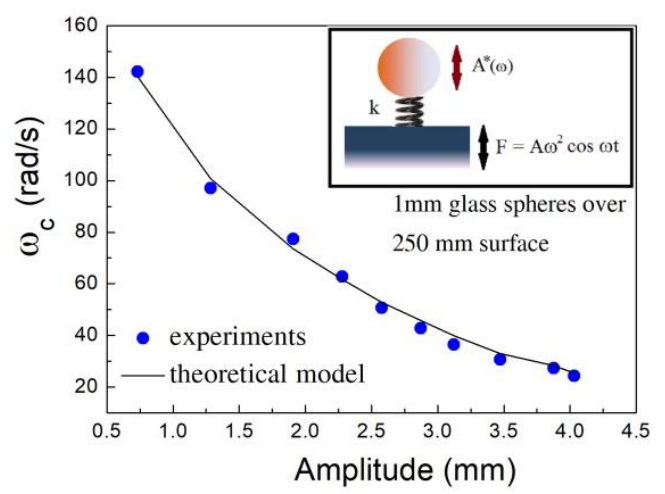

Fig. 6. Comparison between model and experiments.

The agreement is good and the model is able to describe quite well the experimental dependence of the critical frequencies on the input amplitude. As mentioned before and in agreement with other authors $[14,61]$, the idea of a harmonic oscillator resonating at the natural frequency corresponding to the particle material is not adequate and an approach as the one presented here is necessary.

\subsection{Numerical approach}

Using a classical discrete element method model (DEM) to represent the dynamical interaction between the moving particles and the rough surface, the simulation of the vibrating substrate was performed following the two-dimensional formulation in [62] and the three-dimensional extension in [63]. The details of the model can be found in $[55,56]$. Basically, the particles are modelled as spheres using a "soft-particle" approach, where each sphere can have multiple contacts that may persist for extended contact durations (typically during 50 time steps). Normal and tangential forces can develop at the contact point between two spheres. The presence of tensile forces at the contacts models cohesive particles. In this case, short-range interactions occur up to the breaking of the adhesive forces or the severing of the liquid bridge. No longrange interactions are present in this model. A rolling resistance parameter is introduced to represent the effect of the shape of the particle on its rolling capability [64]. Table 2 resumes the values used in the series of simulations for vibrating systems.

Table 2. Simulation parameters

\begin{tabular}{|c|c|}
\hline Parameter & Value \\
\hline Density for glass & $2500 \mathrm{~kg} / \mathrm{m}^{3}$ \\
\hline Friction coefficient & 0.5 \\
\hline Restitution coefficient & $0.4-0.8$ \\
\hline Stiffness (normal direction) & $10^{6} \mathrm{~N} / \mathrm{m}$ \\
\hline Stiffness (tangential direction) & $1.6 \times 10^{6} \mathrm{~N} / \mathrm{m}$ \\
\hline Dashpot constant $\left(\mathrm{b}_{\mathrm{n}}\right)$ & $0.11 \mathrm{~kg} / \mathrm{s}$ \\
\hline Dashpot constant (tangential direction) & $0.4 \mathrm{~b}_{\mathrm{n}}$ \\
\hline Rolling resistance & $0.1-0.3$ \\
\hline Surface tension & $72 \mathrm{mN} / \mathrm{m}$ \\
\hline Hamaker constant & $6.5 \times 10^{-20} \mathrm{~J}$ \\
\hline
\end{tabular}

The results for both simulations, multilayer and monolayer, are presented in what follows. For the first case, a rough surface with $500 \mu \mathrm{m}$ spheres randomly deposited and glued to configure the vibrating platform was used. Then, 200 spheres of $1 \mathrm{~mm}$ diameter were randomly deposited on the surface to consider a large number of different configurations. No collisions were allowed between the moving particles. The rough surface is then vertically oscillated with a given amplitude and frequency emulating the experiments.

Figure 7 shows a comparison of the simulations with the theoretical and experimental results. The exponential decay is recovered in simulations and the agreement with experiments is reasonably good. The lines indicate fitted exponential curves. The difference in the behaviour between simulations and experiments is attributed to the surface roughness. Indeed, the number of layers and the mean distance between spheres in the upper layer of the simulated substrate do not have the topological complexity of the surface generated through real glued spheres. Thus, the ease for the moving beads to overcome the barrier inside a hole is greater in simulations than experiments. This is especially evident for lower amplitudes, where the critical frequencies are lower in simulations. Trials with other ratios confirmed this behaviour.

For the monolayer case, results for two different coverage values for surface $S 1$ are shown in Figure 8. The vertical series of filled symbols defined for two different amplitudes in each figure correspond to the different size ratios used in the numerical model, i.e., moving spheres size/glued spheres size. In Figure 8 (a), the symbols from top to bottom at $A=2 \mathrm{~mm}$ are equal to $1.5,2,3,5,6,7$ and, at $A=4 \mathrm{~mm}$, they are $1.5,2$ and 3 , respectively, and are practically overlapped. In Figure 8 (b), the ratios correspond to 1.5, 2 and 3 for the two values of the amplitude. Recall that the low 
ratio limit cannot be reached in experiments due to the difficulty for detecting the particle movement.

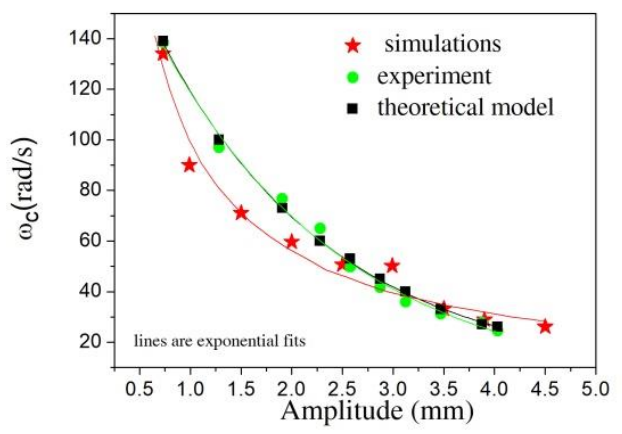

Fig. 7. Comparison of simulations, experiments and theory for the case of $1 \mathrm{~mm}$ spheres over $500 \mu \mathrm{m}$ surface.
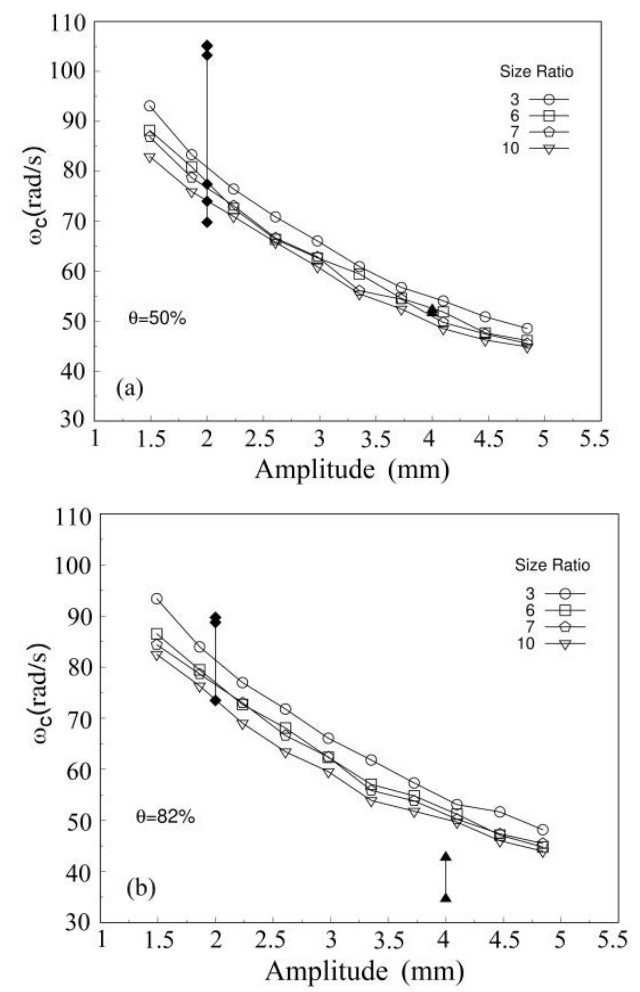

Fig. 8. Comparison for $\omega_{c}$ versus amplitude for S1.

For the case of small amplitudes and low ratios, the frequencies practically overlap, i.e., the particles are trapped inside local holes, especially for the lower coverage. On the other hand, at small amplitudes, the spread out of the frequency values is larger than for larger amplitudes. This is especially evident in Figure 8 (a), where the range of numerical and experimental values at $A=2 \mathrm{~mm}$ is quite larger than for $\mathrm{A}=4 \mathrm{~mm}$. As said before, the effect of the size ratio is more important at lower amplitudes, where the particles get trapped.

A reasonable agreement is found at low amplitudes in Figure 8 (b), but, for the case of high amplitude, simulations overestimate the experimental results. This difference is attributed to possible biased positions of the mobile spheres in the experiments due to the local inhomogeneity in the construction of the surface, especially when approaching to the maximal random close packing $(82 \%)$.
The dependence on coverage was inspected through simulations and Figure 9 shows the behaviour of $\omega_{c}$ with $\theta$ for three different relative amplitudes $A$. It is clear that the incidence of surface coverage is more important when $\theta<60 \%$ at all amplitude values. For that reason, the role of the coverage is weak in the experiments (Figure 6).

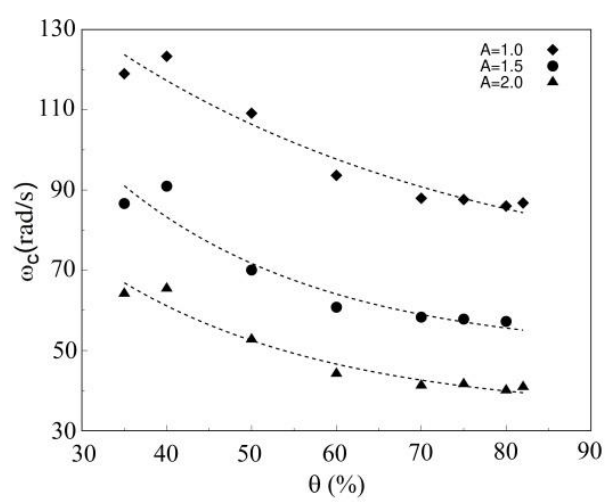

Fig. 9. Frequency $\omega_{\mathrm{c}}$ vs. $\theta$ for different relative amplitudes.

\section{Destabilization by centrifugation}

Finally, in this Section, the movement initiation of millimetre spherical particles on rotating rough surfaces is presented when the external perturbation is a centrifugal force. The experiment main goal is to prove that the sole consideration of the contact forces between the moving spheres and the glued spheres belonging to the rough surface are not sufficient to explain the critical angular velocities found. Not only friction is the obvious answer but also adhesion has to be taken into account when the thermodynamic work of adhesion between surfaces is significant.

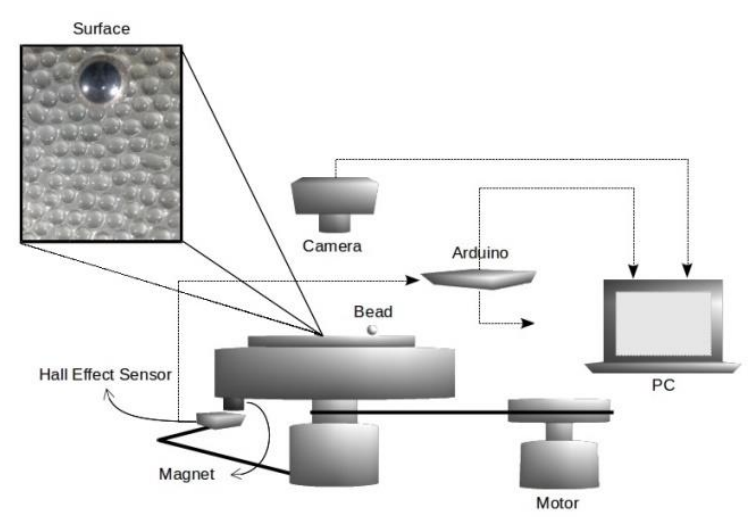

Fig. 10. Sketch of the experimental set up used to destabilize the grains by centrifugation

\subsection{Experimental set up and materials}

The experimental set up is schematized in Figure 10. A rough surface was mounted on a rotating plate whose speed was controlled by an open-hardware Arduino microcontroller. The details can be found in [37]. Initially, 12 spherical grains of the same diameter and material were located on the surface at different distances $r_{0}$ from the disk axis and in positions preventing possible subsequent collisions. The 
experiment was repeated several times, obtaining a total of 15 runs for each distance $r_{0}$. Relative humidity was always less than $50 \%$ to avoid capillary effects.

Two material types were used for the deposited spheres: glass beads (diameter range 3-8 $\mathrm{mm}$ ), and stainless-steel ball bearings (diameter range 4-6 $\mathrm{mm}$ ). The bead sizes were chosen to ensure a good resolution for the grains in the video, which had a frame of $512 \times$ 384 pixels. Two rough surfaces were used. One built by gluing glass beads with mean diameter of $500 \mu \mathrm{m}$ ( $\sim 10 \%$ relative dispersion) and the other with a mean of $1000 \mu \mathrm{m}$ ( $\sim 5 \%$ relative dispersion $)$.

A typical experimental run to determine the critical angular velocity, $\Omega_{c}$, for a given particle deposited on the rotating surface to start its movement, is as follows. Once the particles are set on the surface, the platform started its rotation increasing its velocity every $5 \mathrm{~s}$ with a duty cycle of $0.4 \%$ and until the last particle is detached. All the process is recorded by a digital camera (Casio Exilim EX-ZR400, at $30 \mathrm{fps}$ ) and the time at which each bead started to move on the surface was determined thanks to a video post processing [37]. The criterion to determine whether a particle is in incipient motion is that at least a half of particle diameter displaces from its initial position. The angular frequency for which this event occurs for the first time corresponds to $\Omega_{c}$. In case of an eventual collision between a particle that started its movement and a particle at rest, the detachment of this latter was discarded from the records.

\subsection{Simple modelling vs. experimental results}

Considering the centrifugal force in the destabilization of the particles on the rotating rough surface, basic physics indicates that this force could be derived from the equilibrium equations between the particle and the obstacles presented by the rough surface. Nevertheless, the sole consideration of these obstacles is not enough to explain the experimental results obtained for small particles. Figure 11 illustrates this statement. The curves in the figure correspond to the following equations in horizontal and radial directions:

$$
\begin{gathered}
N \cos \theta-m g=0 \\
N \sin \theta=\frac{m v^{2}}{r_{0}}
\end{gathered}
$$

giving a critical velocity $v_{c}$ :

$$
v_{c}=\left(\operatorname{tg} \theta r_{0} g\right)^{1 / 2}
$$

When the effects of friction are included, we obtain the following:

$$
\begin{gathered}
N \cos \theta-m g-F_{\mu} \sin \theta=0 \\
N \sin \theta+F_{\mu} \cos \theta=\frac{m v^{2}}{r_{0}} \\
v_{c}=\left(\frac{r_{0} g}{\cos \theta-\mu_{r} \sin \theta}\right)^{1 / 2}\left(\sin \theta+\mu_{r} \cos \theta\right)^{1 / 2}
\end{gathered}
$$

Finally, when adhesion forces are considered:

$$
\begin{gathered}
N \cos \theta-m g-F_{\mu} \sin \theta-F_{a} \cos \theta=0 \\
N \sin \theta+F_{\mu} \cos \theta-F_{a} \sin \theta=\frac{m v^{2}}{r_{0}} \\
v_{c}=\left(\frac{r_{0}}{m\left(\cos \theta-\mu_{r} \sin \theta\right)}\right)^{\frac{1}{2}} \times \\
\left(m g\left(\sin \theta+\mu_{r} \cos \theta\right)+\mu_{r} F_{a}\right)^{1 / 2}
\end{gathered}
$$

These equations were derived for the model particle sketched in Figure 12(a). In Figure 12(b), the interaction with only one surface bead immediately before detachment is considered.
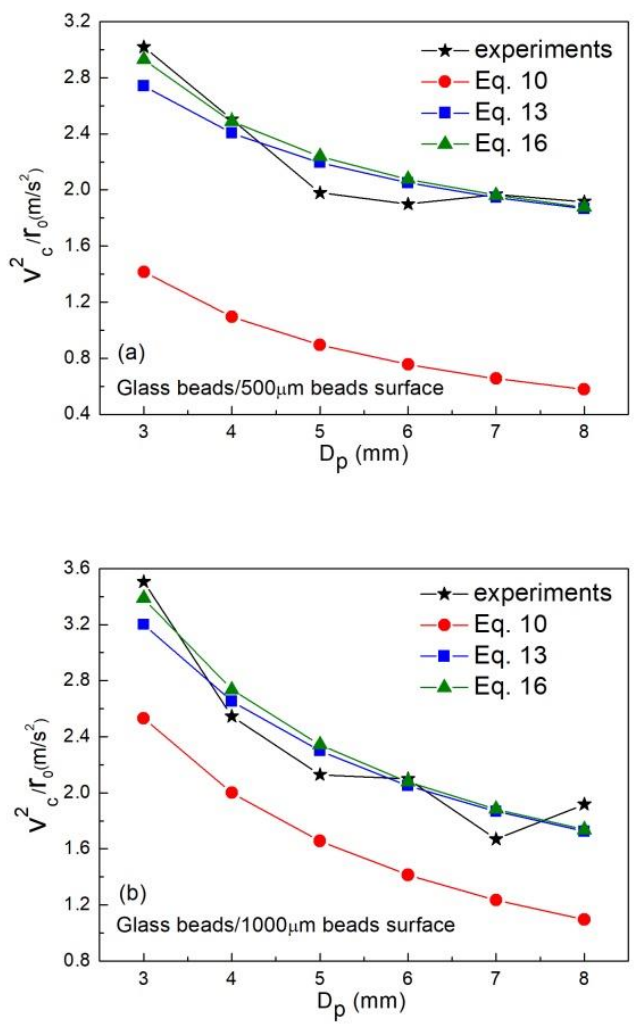

Fig. 11. Experimental and model results for Equations 10, 13 and 16.

The critical velocities, $v_{c}$, given by Equations 10 , 13 and 16 are used to obtain the critical centripetal acceleration at which detachment is achieved, i.e., $v_{c}^{2} / r_{0}$. Figure 11 shows the results vs. particle diameter $D_{p}$. Equations 8 to 10 correspond to the sole consideration of the particle weight and the normal force. Note that the location of the particle in Figure 12 is the most unfavourable one for particle destabilization, i.e., the particle has to overcome the surface bead profile in order to escape. 


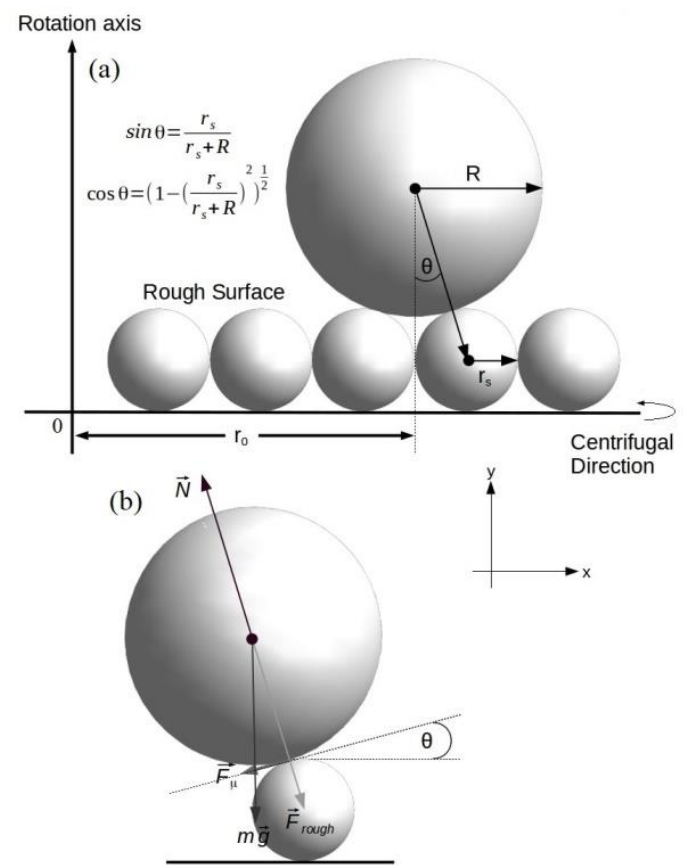

Fig. 12. Force diagrams for the determination of the static equilibrium conditions.

It is evident from Figure 11 (a) and (b) that the theoretical prediction under-estimates the experimental measurements, which correspond to the average over the initial positions of the beads on the rotating surfaces.

Equations 11 to 13 include the rolling friction, $F_{\mu}=\mu_{r} N$, where $\mu_{r}$ is the rolling friction coefficient, taken as 0.13 for glass over glass on the $500 \mu \mathrm{m}$ surface $[55,64]$. It is important to remember that the rolling coefficient of a particle over a flat surface is inversely proportional to its radius, $R_{p}$ (Coulomb's law) and proportional to the reduced radius $\frac{1}{R_{p}}+\frac{1}{r_{a}}$ when the particle rolls over an asperity of radius $r_{a}$ [65]. In the results plotted in Figure 11 (a), $\mu_{r}$ is assumed the same for the particle radii range used here on a given surface. Indeed, it can be easily calculated that the reduced radius changes at most $10 \%$ for the $500 \mu \mathrm{m}$ surface. In contrast, when the size of the beads glued on the rough surface changes, the reduced radius changes by a factor of two, which affects the value of $\mu_{r}$, as shown in Figure $11(\mathrm{~b})$, where $\mu_{r}=0.06$ was considered for the $1000 \mu \mathrm{m}$ surface.

Although the agreement between this second approach and the experiments is good, the predicted values for the smaller grains are lower than expected.

Finally, considering the adhesive forces between the contacting surfaces (Equations 14 and 15), the critical velocity results the one in Equation 16. Among the many theoretical models to evaluate the adhesion forces between surfaces, the one developed by Goldasteh and co-workers was considered here [6]. It is out of the scope of the present work to review those models and only the introduction of the adhesion force used in the present approach is performed in what follows. Interested readers can look for further information in the references $[6,37,52,57,66]$.
By extending the formalism of the JKR theory [52] to a rough contact area, Goldasteh et al. [6] reformulated the pull-off force needed to overcome adhesion on a rough surface for a particle with diameter $D_{p}$ as:

$$
\mathrm{F}_{a}=\frac{\mathrm{D}_{\mathrm{p}}}{4} 0.0029 \mathrm{~W}_{a}\left[1.5 \pi^{2} \mathrm{e}^{-\frac{0.6}{\Delta \mathrm{C}^{2}}}\right]^{3}
$$

$W_{a}$ is the thermodynamic work of adhesion interaction at the particle-surface contact. When the particle is in contact with another particle of the surface (not a flat surface), as in the present case, $D_{p}$ must be replaced by a reduced diameter. The parameter $\Delta \mathrm{C}$ is defined as the ratio between the elastic extension of each asperity on the surface before separation and the root mean square of the asperity heights. In this way, Equation (17) gives the adhesion force used in Equations 14 to 16, with $D_{p}$ replaced by the corresponding reduced diameter.

Figure 11 shows the improvement of the description of the experimental data. The effect of the adhesion force is most appreciable for the smaller grains, while for the larger ones, the theoretical data points practically overlap and the adhesion effect is almost negligible. $W_{a}$ is equal to $0.40 \mathrm{~J} / \mathrm{m}^{2}$ for glass over glass [42]. The literature reports that $\Delta \mathrm{C}$ ranges from 0.5 to $0.95[12,13]$. Here, 0.92 and 0.95 are the values for the best fits of the experimental data in Figure 11 (a) and (b), respectively.

Figure 13 shows the results for stainless steel grains on both surfaces. In this case, $W_{a}$ equals $0.15 \mathrm{~J} / \mathrm{m}^{2}$, which means a decrease by a factor of almost 2.7 . Consequently, the adhesion effects become smaller, as evidenced by the figure.

For this material combination, the sole consideration of the forces in Equations 11 and 12 is enough to describe the results. The values of $\mu_{r}$ in this case are 0.040 and 0.015 , for $500 \mu \mathrm{m}$ and $1000 \mu \mathrm{m}$, respectively. As expected, these values are smaller than those for the glass-glass interaction. As before, the reduction in $\mu_{r}$ results from the change in size of the glued beads on the surface. The best value for $\Delta \mathrm{C}$ resulted to be 0.92 for both cases.

In Figure 13, the results of Equations 10, 13 and 16 are close to each other and to the experiments. The lower $W_{a}$ and rolling friction make this scenario closer to the ideal one where only the weight and the normal are considered. A comparison of the behavior of glass particles with that of stainless-steel particles shows that the increase in the critical detaching velocities for glass comes from friction and adhesion effects.

\section{Final remarks}

The dynamics of the movement initiation of fine and small particles in different scenarios has been reviewed in the previous sections.

For fine particles (microns), implementation of a Kinetic Monte Carlo methodology based on the stochastic nature of the resuspension phenomenon and its straight analogy with desorption of particles from heterogeneous surfaces made it possible to develop a 
model which reasonably depicts the main features of particle re-entrainment dynamics. The three principal mechanisms enounced in the literature as the possible responsible for the movement initiation of particles on a surface and subjected to wind, can be easily formulated through KMC.
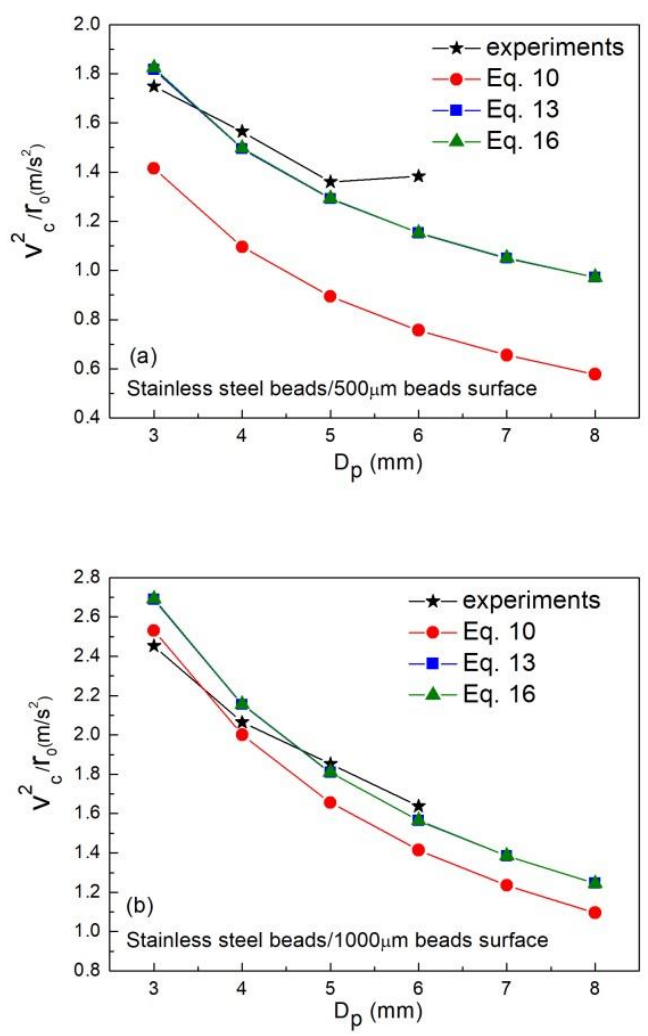

Fig. 13 Experimental and model results.

The concept of resuspension as an activated problem where the role of temperature is played here by the turbulent air flow, provides a vision from the physical foundations of statistical mechanics, which complements and enriches the understanding of particle resuspension. It also enables to develop new techniques as the so called KPR (Kinetic programed Resuspension) that, like its thermodynamic counterpart Temperature Programmed Desorption (TPD), could be used to get important information about the characteristics of the surface where resuspension is taking place [67].

It is important to point out that the model presented in the first part of this work can be extended to hydrodynamic forces in directions different than the parallel to the plane one, with a suitable re-definition of the intervening forces and resuspension rates. In the same way, as soon as the intervening forces are known, smaller particles can be modelled up to $1 \mu \mathrm{m}$ [68].

The whole set of experiments for vibration of small particles (millimetre scale) reveals that the incipient movement initiates at critical frequencies that decrease exponentially with the amplitude of oscillations. These frequencies are much lower than those related to the elastic properties of the particle material $\left(6 \times 10^{6} \mathrm{rad} / \mathrm{s}\right.$ or higher). Thus, the idea of a harmonic oscillator resonating at a natural frequency related to the particle material, alone, is inadequate. The theoretical model proposed describes the experimental behaviour quite closely by assuming that the entire moving particle interactions with the surface are represented by the stiffness of the vibrating spring, resulting in a lower value compared with the one derived from glass stiffness alone.

In this size range, DEM simulations offer a good description of particle movement initiation. The exponential decay is numerically recovered and the role of the particle ratio and the surface coverage on the critical frequency is captured by the model. Nevertheless, the lack of a real topological description of the surface roughness is responsible for the lowerthan-expected critical frequencies.

On the other hand, the force required to detach a particle on a rotating rough surface is dependent on its size and on the size of the surface roughness, as demonstrated in the experiments of the preceding section. They also prove that the consideration of obstacle geometry, and even friction, is insufficient to explain the results for glass particles, although in the range of small particles. It is also demonstrated that the adhesive force contribution could be significant when those particles are involved and their thermodynamic work of adhesion is sufficiently high, for example, in metals such as tungsten, iron or copper, where the works of adhesion exceed $1 \mathrm{~J} / \mathrm{m}^{2}$ [57]. This is particularly interesting when force models are sought in the formulation of numerical simulations.

It is important to note that the phenomenon of resuspension of fine particles and the incipient motion of small particles are usually treated as separate problems, commonly sharing different points of view. Nevertheless, many concepts and approaches could be exploited if a global viewpoint on the dynamic of the particles and, on the stochastic nature of the particlesurface interactions, considered at the same time.

For example, attractive forces not coming from capillary effects but those due to adhesion interactions are not always taken into account when micromechanical models of small particles (of the order of $1 \mathrm{~mm}$ ) are developed. This was discussed in Section 4, where particle destabilization by centrifugation was analysed.

On the other hand, when fine particles interact with rough surfaces, not only adhesion forces have to be modified to account for roughness effects but also the geometry of the contacts becomes crucial when the size of the surface protuberances becomes comparable to the size of the deposited particles, and the moment balance of adhesive forces starts to be affected [69]. This scenario turns to be very complex for a classical mechanical approach and the aerosol science community would be benefit from adding a statistical mechanical approach to the interactions of fine particles with surfaces.

As a final remark, the present work has reviewed different phenomena where an external perturbation causes movement initiation of a particle on a surface. Important evidence about the type of the interactions between particle and surface can be obtained from it. 
No matter the size of the particles involved, the different phenomena could be treated under a global multi-scaled view, using a combination of all the models here reviewed, to find the limits where a change in the size of the particles can or cannot affect the problem under study.

Authors want to acknowledge to Universidad Nacional de San Luis and CONICET for financial support through respective projects PROICO 03-2718 and PIP $\mathrm{N}^{\circ}$ $11220170100245 \mathrm{CO}$. International collaborations were supported through ECOS-SUD-MINCyT A15E03 and LIA Franco-Argentin, Physique et Mécanique de Fluides.

\section{References}

[1]. J. Csavina, J. Field, M.P. Taylor, M. Gao, S Landzuri, A. Betterton, A.E. Sáez, Sci. of the Tot. Environ. 433, 58 (2012)

[2]. M. Stovern, O. Felix, J. Csavina, K. Rine, M.R. Russell, R. Jones, R.M. Jones, M. King, E.A. Betterton, A.E. Sáez, Aeolian Res. 14, 75 (2014)

[3]. D.S. Bisquert, J.M.P. Castejón, G.G. Fernández, Atmos. Environ. 152, 256 (2017).

[4]. A. Rondeau, S. Peillon, S., A. Roynette, J.C. Sabroux, T. Gelain, F. Gensdarmes, V. Rohde, C. Grisolia, E. Chassefière, J. Nucl. Mat., 463, 873 (2015)

[5]. T. Zhang, S. Yu, Q. Sun, W. Peng, J. Wang, Prog. Nucl. Energy, 98, 313 (2017)

[6]. I. Goldasteh, A. Goodarz, A. Ferro, J. Aerosol Sci. 66, 62 (2013)

[7]. E. Rabinovich, H. Kalman, Powder Technol., 192, 318 (2009)

[8]. E. Rabinovich, H. Kalman, Powder Technol., 192, 326 (2009)

[9]. M.W. Reeks, J. Reed, D. Hall, J. Phys. D: Appl. Phys. 21, 574 (1988)

[10]. M.W. Reeks, D. Hall, J. Aerosol Sci. 32, 1 (2001)

[11]. Y. Shimada, M. Sunada, M. Mizuno, Y. Yonezawa, H. Sunada, M. Yokosuka, H. Kimura, H. Takebayashi, Drug Dev. Ind. Pharm. 26, 149 (2000)

[12]. M. Soltani, G. Ahmadi, J Adhes Sci Technol. 8, 763 (1994)

[13]. M. Soltani, G. Ahmadi, J. Adhes. 44, 161 (1994)

[14]. G. Ziskind, M. Fichman, C. Gutfinger, J. Aerosol Sci. 31, 703 (2000)

[15]. K. Hein, T. Hucke, M. Stintz, S. Ripperger, Part. Part. Syst. Charact 19, 269 (2002)

[16]. S. Ripperger, K. Hein, China Particuology 3, 3 (2005)

[17]. N. Tippayawong, N., I. Preechawuttipong, Aust. J. Basic \& Appl. Sci. 5, 356 (2011)

[18]. M.B. Ranade, Aerosol Sci. Technol. 7, 161 (1987)
[19]. W.T. Leung, S.C. Fu, G.N. Sze To, C.Y.H. Chao, Aerosol Sci. Technol. 47, 1239 (2013)

[20]. T.T. Nguyen, C. Rambanapasi, A.H. de Boer, H.W. Frijlink, v.D.P.M. Ven, J. de Vries, H.J. Busscher, v.D.K. Voort Maarschalk, Int. J. Pharm. 393, 88 (2010)

[21]. G.R. Salazar-Banda, M.A. Felicetti, J.A.S. Gonçalves, J.R. Coury, M.L. Aguiar, Powder Technol. 173, 107 (2007)

[22]. X. Meng, F. Jia, W. Qiu, Y. Han, Y. Zeng, Y. Xiao, P. Chen, Powder Technol. 348,1 (2019)

[23]. P. Van Liedekerke, E. Piron, J. Vangeyte, S. Villette, H. Ramon, E. Tijskens, Granul. Matter 10, 247 (2008)

[24]. X.-D Ma, Y.-B Zhang, Y. Liu, X.-W. Zheng, Granul. Matter 18, 8 (2016)

[25]. L. Oger, A. Valance, Eur. Phys. J. Web Conf. 140, 12004 (2017)

[26]. C.-C. Liao, S.-S. Hsiau, KONA Powder Part. J. 33, 109 (2016)

[27]. C R K Windows-Yule, New J. Phys. 18, 033005 (2016)

[28]. C. Morize, E. Herbert, A. Sauret, Phys. Rev. E 96, 032903 (2017)

[29]. P. Eshuis, K. van der Weele, D. van der Meer, R. Bos, D. Lohse, Phys. Fluids 19, 123301 (2007)

[30]. S. Renard, T. Schwager, T. Pöschel, C. Salueña, Eur. Phys. J. E 4, 233 (2001)

[31]. Y. Mawatari, T. Koide, Y. Tatemoto, S. Uchida, K. Noda, Powder Technol. 123, 69 (2002)

[32]. G. D’Anna, G. Gremaud, Phys. Rev. E 64, 011306 (2001)

[33]. F. Melo, P.B. Umbanhowar, H.L. Swinney, Phys. Rev. Lett. 75, 3838 (1995)

[34]. F. Melo, P.B. Umbanhowar, H.L. Swinney, Phys. Rev. Lett. 72, 172 (1994)

[35]. T.H. Metcalf, J.B. Knight, H.M. Jaeger, Phys. A 236, 202 (1997).

[36]. M. Hubert, H. Kalman, Granul Matter 6, 159 (2004)

[37]. K.A. Valenzuela Aracena, R.O. Uñac, I. Ippolito, A.M. Vidales, Particuology 53, 92 (2020)

[38]. M.E. Mullins, L.P. Michaels, V. Menon, B. Locke, M.B. Ranade, Aerosol Sci. Technol. 17, 105 (1992)

[39]. R. Fuchs, T. Weinhart, J. Meyer, J., H. Zhuang, T. Staedler, X. Jiang, S. Luding, Granul. Matter 16, 281 (2014)

[40]. F. Soepyan, S. Cremaschi, B. McLaury, C. Sarica, H. Subramani, G. Kouba, H. Gao, Powder Technol. 292, 272 (2016)

[41]. C. Henry, J.-P. Minier, Prog. Energy Combust. Sci. 45,1 (2014)

[42]. A. Ibrahim, P. Dunn, R. Brach, J. Aerosol Sci. 29, 765 (2003)

[43]. F. Zhang, M. Reeks, M. Kissane, J. Aerosol Sci. 58, 103 (2013) 
[44]. J.G. Benito, K.A.Valenzuela Aracena, R.O. Uñac, A.M. Vidales, I. Ippolito, J. Aerosol Sci. 79, 126 (2015)

[45]. J.G. Benito, R.O. Uñac, A.M. Vidales, I. Ippolito, J. Aerosol Sci. 100, 26 (2016)

[46]. V.P. Zhdanov, Dynamics of Molecular Processes on Surfaces. In: Elementary Physicochemical Processes on Solid Surfaces, Fundamental and Applied Catalysis (Springer, Boston, 1991).

[47]. H. Wen, G. Kasper, J. Aerosol Sci. 20, 483 (1989)

[48]. E.M. Hughes, The chemical statics and kinetics of solutions (Academic Press, London, 1971)

[49]. K. Binder, D. Heermann, Monte Carlo simulation in statistical physics: An introduction (Springer Verlag, Berlin, 1992)

[50]. K.A. Fichthorn, W. H. Weinberg, J. Chem. Phys. 95, 1090 (1991)

[51]. J. Sales, R. Uñac, M. Gargiulo, V. Bustos, G. Zgrablich, Langmuir 12, 95 (1996)

[52]. K.L. Johnson, K. Kendall, A.D. Roberts, Proceedings of the Royal Society of London A. Mathematical and Physical Sciences 324, 301 (1971)

[53]. M. Guingo, J.P. Minier, J. Aerosol Sci. 39, 957 (2008)

[54]. A.H. Ibrahim, P.F. Dunn, M.F. Qazi, J. Aerosol Sci. 39, 645 (2008)

[55]. K.A. Valenzuela Aracena, J.G. Benito, L. Oger, I. Ippolito, R.O. Uñac, A.M. Vidales, Particuology 40, 1 (2018)

[56]. K.A. Valenzuela Aracena, L. Oger, R.O. Uñac, A.M. Vidales, Granul. Matter 21, 51 (2019)

[57]. J.N. Israelachvili, Intermolecular and surface forces (Academic Press, London, 1991)

[58]. H.P. Zhu, Z.Y. Zhou, R.Y. Yang, A.B. Yu, Chem Eng. Sci. 62, 3378 (2007)

[59]. S. Herminghaus, Adv. Phys. 54,221 (2005)

[60]. I. Kovacic, J. Sound Vib. 330, 4313 (2011)

[61]. T. Dabros, P. Warszynsky, T.G.N. van de Ven, J. Colloid Interf. Sci. 162, 254 (1994)

[62]. S. B. Savage, Disorder, diffusion, and structure formation in granular flows. In: Disorder and granular media (D. Bideau Ed., Amsterdam: North Holland, 1993, p.264)

[63]. L. Oger, I. Ippolito, A.M. Vidales, Granul. Matter 9, 267 (2007)

[64]. J. Ai, J.-F. Chen, J.M. Rotter, J.Y. Ooi, Powder Technol. 206, 269 (2011)

[65]. R. Cross, Eur. J. Phys. 36, 065029 (2015), and references there in.

[66]. B. V. Derjaguin, V. M. Muller, Y.P. Toporov, J. Colloid Interf. Sci. 53, 314 (1975)

[67]. J.G. Benito, R.O. Uñac, A.M. Vidales, I. Ippolito, J. Aerosol Sci. 122, 21 (2018)
[68]. A. Rondeau, S. Peillon, A.M. Vidales, J. Benito, R. Uñac, J.-C. Sabroux, F. Gensdarmes, J. Aerosol Sci. 154, 105735 (2021).

[69]. Y. Jiang, S. Matsusaka, H. Masuda, Y. Qian, Powder Technol. 186, 199 (2008) 